\title{
The Einstein polarization interferometer for cosmology (EPIC) and the millimeter-wave bolometric interferometer (MBI)
}

\author{
P.T. Timbie ${ }^{\mathrm{a}, *}$, G.S. Tucker ${ }^{\text {b }}$, P.A.R. Ade ${ }^{\mathrm{c}}$, S. Ali ${ }^{\mathrm{d}}$, E. Bierman ${ }^{\mathrm{e}}$, E.F. Bunn ${ }^{\mathrm{f}}$,
} C. Calderon ${ }^{\text {c }}$, A.C. Gault ${ }^{\text {a }}$, P.O. Hyland ${ }^{\text {a }}$, B.G. Keating ${ }^{\text {e }}$, J. Kim ${ }^{\text {b }}$, A. Korotkov ${ }^{\text {b }}$, S.S. Malu ${ }^{\mathrm{a}}$, P. Mauskopf ${ }^{\mathrm{c}}$, J.A. Murphy ${ }^{\mathrm{g}}$, C. O'Sullivan $^{\mathrm{g}}{ }^{\mathrm{a}}$, L. Piccirillo ${ }^{\mathrm{c}}$, B.D. Wandelt ${ }^{\mathrm{h}}$

\author{
${ }^{a}$ Department of Physics, UW-Madison, Madison, WI 53706, USA \\ ${ }^{\mathrm{b}}$ Department of Physics, Brown University, Providence, RI 02912, USA \\ ${ }^{\mathrm{c}}$ Department of Physics and Astronomy, Cardiff University, Cardiff CF24 $3 Y$ Y, Wales, UK \\ d Advanced Detector Group, LLNL, Livermore, CA 94550, USA \\ ${ }^{\mathrm{e}}$ Department of Physics and Astronomy, University of California, San Diego, CA 92093-0424, USA \\ ${ }^{\mathrm{f}}$ Physics Department, University of Richmond, Richmond, VA 23173, USA \\ ${ }^{g}$ Department of Experimental Physics, National University of Ireland, Maynooth, Co. Kildare, Ireland \\ ${ }^{\mathrm{h}}$ Department of Astronomy and Department of Physics, University of Illinois, Urbana, IL 61801, USA
}

Available online 13 November 2006

\begin{abstract}
We provide an overview of a mission concept study underway for the Einstein Inflation Probe (EIP). Our study investigates the advantages and tradeoffs of using an interferometer $(E P I C)$ for the mission. We also report on the status of the millimeter-wave bolometric interferometer (MBI), a ground-based pathfinder optimized for degree-scale CMB polarization measurements at $90 \mathrm{GHz}$. (c) 2006 Elsevier B.V. All rights reserved.
\end{abstract}

PACS: 95.75.Kk; 95.75.Hi; 95.85.Bh; 95.85.Fm; 98.80.Es; 98.80.-k; 98.70.Vc

Keywords: Einstein inflation probe; Cosmic microwave background; Polarization; Interferometry

\section{Contents}

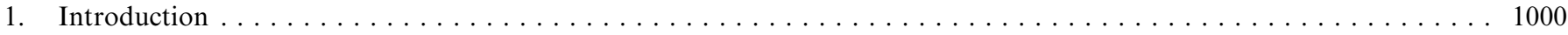

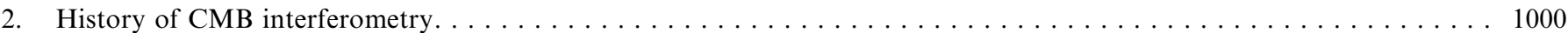

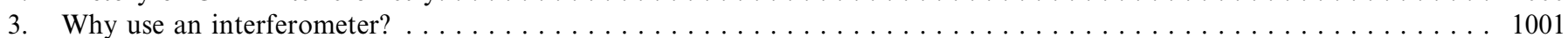

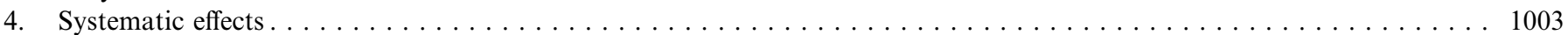

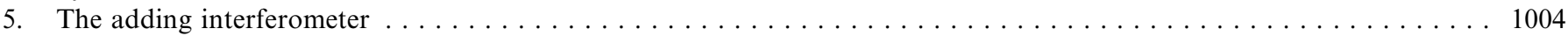

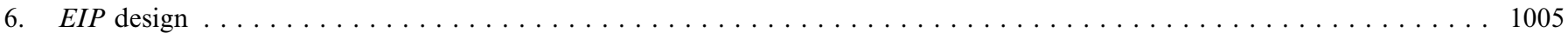

7. The millimeter-wave bolometric interferometer $(\mathrm{MBI}) \ldots \ldots \ldots \ldots \ldots \ldots \ldots$

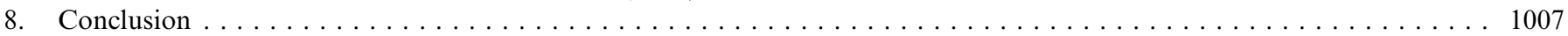

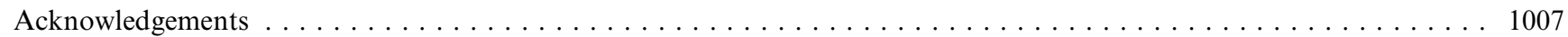

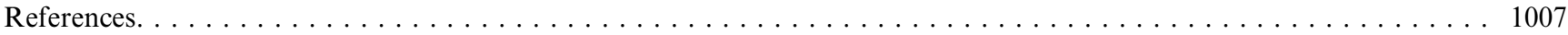

\footnotetext{
* Corresponding author. Fax: +1 6082630361.

E-mail address: pttimbie@wisc.edu (P.T. Timbie).
} 


\section{Introduction}

During this conference we heard the latest results from $W M A P$ (Page et al.). WMAP sets an upper limit on $B$-modes at large angular scales that constrains the tensor to scalar ratio to $T / S<0.3$. Furthermore, both $W M A P$ and Boomerang (MacTavish et al., 2003) suggest that the scalar spectral index is different from unity, which implies that $T / S$ may be as large as 0.1 and raises the level of enthusiasm in the field. Although the anticipated gravitational-wave CMB polarization signals are very weak, arrays of $\sim 1000$ detectors that can be built today have enough raw sensitivity to detect $B$-modes at a level of $T / S=0.01$ with integration times of $\sim 1$ year. The greater challenge is to design a mission with unprecedented control of systematic effects. Starting in 2004 three groups began NASA-supported mission concept studies for the Einstein Inflation Probe $(E I P)$. In this paper we describe the goals and status of the study undertaken by one of these groups. Our concept focuses on understanding the advantages and disadvantages of using interferometry in the EIP. We call the concept the Einstein Polarization Interferometer for Cosmology (EPIC).

Interferometers have been used for many years for studying the CMB temperature and polarization power spectra and the Sunyaev-Zel'dovich effect. In fact, the first detection of CMB $E$-mode polarization was made by an interferometer: DASI (Kovac et al., 2002). There are many reasons to consider using interferometers for CMB measurements in the future, particularly for measurements of the primordial B-mode signal from gravitational waves. The key reason is to control systematic effects. The interagency Task Force for CMB Research (Bock et al.) describes how the EIP might be designed to overcome the challenges to measuring the $B$-mode signal. Interferometry offers one option. However, in order to achieve the goals of the EIP using an interferometer, several technolog- ical challenges will have to be overcome. Some of these are different from the challenges facing an imaging approach to the EIP, while many are similar, such as the need for millimeter-wave detector arrays and schemes to read them out. We outline here a possible implementation. The EPIC mission concept study is closely coupled with our development of a ground-based demonstration of some of the required technologies in the millimeter-wave bolometric interferometer (MBI) (Korotkov et al., 2006).

\section{History of CMB interferometry}

Interferometers have proved to be powerful tools for CMB observations in the past (Table 1). The Sunyaev-Zel'dovich effect has been imaged by the Ryle (Birkinshaw, 1999), OVRO and BIMA interferometers (Carlstrom et al., 2002) and the new SZA (Loh et al., 2005) at centimeter wavelengths. The CMB temperature anisotropy has been imaged by the CAT (Baker et al., 1999), VSA (Dickinson et al., 2004), DASI (Halverson et al., 2002) and CBI (Readhead et al., 2004) interferometers, also at centimeter wavelengths. DASI was the first instrument to detect the CMB polarization (Kovac et al., 2002; Leitch et al., 2005) and CBI has detected CMB polarization at smaller angular scales (Readhead et al., 2004; Cartwright et al., 2005).

Recently, several groups have studied the possibility of building future interferometers specifically to search for small polarization signals in the CMB. Compared to existing interferometers, these new instruments would have to (1) collect more modes of radiation from the sky and (2) operate over a broader range of frequencies, at least up to $90 \mathrm{GHz}$, to be able to detect and reject astrophysical foreground sources. The approaches we are aware of are of two types. (To our knowledge, the details from these studies have not yet been published.) One is to use coherent receivers and increase the number of antennas and receiv-

Table 1

CMB Interferometers from the past, present, and future

\begin{tabular}{|c|c|c|c|c|c|}
\hline Instrument & Location & $N_{\text {Ant }}$ & Frequency $(\mathrm{GHz})$ & $l$-Range & Status \\
\hline ALMA (http://www.alma.nrao.edu) & Atacama & 50 & $30-1000$ & huge & Future \\
\hline AMiBA (http://www.asiaa.sinica.edu.tw/amiba/) & Mauna Loa & 19 & 90 & $120-5000$ & Current \\
\hline ATCA (Subrahmanyan et al., 2000) & Australia & 6 & 9 & $3500-5780$ & Current \\
\hline CARMA (Woody et al., 2004) & Cedar Flat & 23 & $26-230$ & $2400-10^{7}$ & Present \\
\hline CAT (Baker et al., 1999) & UK & 3 & $13-17$ & $339-722$ & Past \\
\hline CBI (Readhead et al., 2004) & Atacama & 13 & $26-36$ & $630-3500$ & Past \\
\hline MINT (Fowler et al., 2005) & Atacama & 4 & 140 & $1200-1900$ & Present \\
\hline OVRO (Leitch et al., 2000) & OVRO & 9 & 30 & $360-2000$ & Past \\
\hline Ryle (Jones et al., 2005) & UK & 8 & 5,15 & $4000-8000$ & Present \\
\hline SZA (Loh et al., 2005) & Cedar Flat & 8 & $26-36,85-115$ & $2400-80,000$ & Present \\
\hline T-W (Timbie and Wilkinson, 1990) & Saskatoon & 2 & 43 & $20-100$ & Past \\
\hline VLA (Partridge et al., 1997) & Socorro & 27 & $5,8,15$ & $5000-8000$ & Present \\
\hline
\end{tabular}

All use coherent receivers and all use HEMT amplifiers, with the exception of ALMA and MINT which use SIS receivers to operate above 90 GHz. 
ers to hundreds. There are several challenges to such an approach. One of the most significant is the construction of a wideband correlator that can handle the additional baselines. DASI and CBI, like other radio interferometers, first amplify the RF signals from each of the $N$ antennas, downconvert in frequency with a mixer, and then split them $N-1$ ways. The correlator then combines these signals in a pairwise fashion to measure visibilities for $N(N-1) / 2$ baselines. For DASI and CBI $N=13$ and the number of baselines is 78 . But for say, $N=100$, there would be 4950 baselines and this type of correlator is not yet feasible (although correlators could be made to correlate only a fraction of the possible baselines). Another challenge is extension of the receivers to frequencies above $30 \mathrm{GHz}$ in order to control foreground signals.

An alternate approach is to use incoherent detectors (bolometers) in an interferometer. Bolometers have the advantage of operating over the entire range of millimeter wavelengths of interest for CMB studies. In addition, they have comparable sensitivity to coherent receivers below $90 \mathrm{GHz}$ and better sensitivity above $90 \mathrm{GHz}$. The high-frequency sensitivity advantage improves in low background environments (balloons and space). Because there are no amplifiers, the main challenge to this approach is combining the signals from the multiple antennas without sacrificing signal-to-noise. A scheme in which the signals are divided $N-1$ ways and combined pairwise would produce extremely low signal levels at each detector and would require $N(N-1) / 2$ extremely sensitive bolometers.

We are considering an alternate beam combination scheme that avoids the problem of large numbers of bolometers and low signal levels. In this approach the signals from an array of $N$ close-packed, circular corrugated horn antennas are coupled to each of $N$ bolometers simultaneously. The beam combiner is a Fizeau combiner (or interferometer) as opposed to the pairwise beam combiner, which is similar to a Michelson stellar interferometer. The signals from redundant baselines add together to improve the signal-to-noise ratio at each bolometer compared to the pairwise combination case. The signals reaching each bolometer are multiplexed in such a way that a portion of the visibility of each baseline appears at each bolometer. When the signals are combined the resultant sensitivity is comparable to that of a filled-dish with an array of bolometers coupled to the same number of modes $(N)$ on the sky.

The scheme we are studying for EIP actually interferes antenna signals in two modes of operation. In one mode, we interfere signals from different antennas to measure the visibility for each baseline. Each visibility selects a narrow range of $l$ values and has no response to very low monopoles. In the second mode, we interfere signals from each antenna with other signals from the same antenna (autocorrelation) to form a correlation polarimeter. This latter mode has lower angular resolution than the first, but can measure large spatial features (low-l). Both modes operate simultaneously to measure the CMB power spectrum from $l=2$ to 600 with a full-sky observation as planned for EIP. This range of $l$ space is dominated by the gravitational lensing signal at high $l$ and includes both the reionization bump at $l \sim 10$ and the peak at $l \sim 100$ due to primordial tensor modes.

\section{Why use an interferometer?}

Why build an interferometer instead of a more traditional imaging system for studying CMB polarization? There are a number of reasons that have motivated the construction of the interferometers listed in Table 1 . The main reason is to control systematic effects, which in some cases are more manageable than in imaging systems. There are additional factors, especially aperture size, that favor interferometric approaches over imaging for space-based systems. For equivalent angular resolution, an interferometer can be substantially simpler and less costly than a single aperture.

Angular resolution. For a monolithic dish of diameter, $D$, equal to the length of a two-element interferometer baseline, $B$, the interferometer has angular resolution (fringe spacing) roughly twice as good as that of the monolithic dish. The reason for this difference in angular resolution is that the filled dish is dominated by spacings that are much smaller than the aperture diameter. The full width to the first zero for a uniformly illuminated aperture of diameter $D$ is $2.4 \lambda / D$. The full width to the first zero for a twoelement interferometer, when the baseline $B$ is much larger than the individual aperture diameter, is $\lambda / B$. It is helpful to consider the difference between the systems in $l$-space as well. For an interferometer the window function peaks at $l=2 \pi B / \lambda$. For an imaging system with a Gaussian beam the window function is $W_{l}=\mathrm{e}^{-l^{2} \sigma^{2}}$. The beamwidth $\sigma=0.42$ FWHM and FWHM $=\left(1.02+0.0135 T_{\mathrm{e}}\right) \lambda / D$ where $T_{\mathrm{e}}$ is the edge taper of the antenna in $\mathrm{dB}$ (Goldsmith, 1998). For an edge taper of $40 \mathrm{~dB}$ (typical for CMB instruments), $\mathrm{FWHM}=1.51 \lambda / D, \sigma=0.66 \lambda / D$ and the window function falls to $10 \%$ of its peak value at $l=2.29 D / \lambda$, which is less than half of the peak $l$-value for an interferometer baseline of the same size.

This angular resolution factor is important because the size of the aperture is a cost-driver for the EIP mission. Angular resolution is important for $\mathrm{CMB}$ polarization measurements in two ways. First, imperfections in the shape and pointing of beams couple the CMB temperature anisotropy into false polarization signals. These problems can be reduced significantly if the CMB is smooth on the scale of the beam size, which happens for beams smaller than $\sim 10^{\prime}$ (Hu et al., 2003). Second, removing contamination of the tensor $B$-mode signal by $B$-modes from weak lensing requires maps of the lensing at higher angular resolution than the scale at which the tensor $B$-modes peak (Knox and Song, 2002).

No rapid chopping and scanning. Imaging systems with either coherent or incoherent detectors typically use some form of "chopping," either by nutating a secondary mirror or by steering the entire primary at a rate faster than the $1 / f$ 
noise in the atmosphere and detectors. Similar approaches are used with arrays of detectors. Since an interferometer does not require this rapid chopping, the time constants of the bolometers used can be relatively long. When using an imaging system to form a two-dimensional (2D) map with minimal striping or other artifacts, the scan method must move the beam (or beams) on the sky at a rapid rate. Interferometers provide direct $2 \mathrm{D}$ imaging and do not require such scanning strategies. In the interferometer, only correlated signals are detected, so it has reduced sensitivity to changes in the total power signal absorbed by the detectors (White et al., 1999).

Clean optics. The simplicity of an interferometric optical system eliminates numerous systematic problems that plague any imaging optical system. Instead of a single reflector antenna, the interferometers we have studied use arrays of corrugated horn antennas. These antennas have extremely low sidelobes and have easily calculable, symmetric beam patterns. Furthermore, there are no reflections from optical surfaces to induce spurious instrumental polarization, an unavoidable problem for any system with imaging optics (Carretti et al., 2001; Carretti et al., 2004). In principle, one could construct an imaging instrument without reflective optics; an array of horn antennas, each coupled directly to a polarimeter, could view the sky directly. Each horn aperture would be sized to provide the required angular resolution. However, such a system uses the aperture plane inefficiently. A single horn antenna in such an imaging system will have angular resolution $\sim 2 \lambda / D$, where $D$ is the horn diameter. An $N-$ element interferometric horn array that achieves the same angular resolution will have a maximum baseline length of $B=D$ (and require the same aperture size), but will collect $N$ modes of radiation from the sky and hence be more sensitive.

Another advantage over an imaging system is the absence of aberrations from off-axis pixels: all feed elements are equivalent for the interferometer. In contrast to an imaging system, the field-of-view (FOV) of an interferometer is determined by the primary beamwidth of the array elements, not by beam distortion and cross-polarization at the edge of the focal plane. One can choose to increase the sensitivity of the instrument by collecting more modes (optical throughput) of radiation from the sky. In the interferometer this can be done by adding additional antennas; the only limitation is the size of the aperture plane rather than optical aberrations in the focal plane. The largest usable FOV for an off-axis Gregorian reflector is approximately $7^{\circ}$ (Hanany and Marrone, 2002). See Table 2 for a comparison of imaging and interferometric optical systems.

Direct measurement of Stokes parameters. Interferometry solves many of the problems related to mismatched beams and pointing errors raised by $\mathrm{Hu}$ et al. (2003); $\mathrm{Hu}$ et al. (2003). This advantage arises because interferometers measure the Stokes parameters directly, without differencing the signal from separate detectors.
Table 2

Comparison of various optical designs for the $E I P$

\begin{tabular}{llllc}
\hline Instrument & $\begin{array}{l}\text { Angular resolution } \\
(\mathrm{FWHM})\end{array}$ & $\begin{array}{l}\text { FOV } \\
\left({ }^{\circ}\right)\end{array}$ & $\begin{array}{l}\text { Aperture } D \\
(\mathrm{~cm})\end{array}$ & Modes \\
\hline $\begin{array}{c}\text { Gregorian } \\
\text { telescope }\end{array}$ & $1.51 \lambda / D$ & $\sim 7$ & 26 & 49 \\
$\begin{array}{c}\text { Imaging horn } \\
\text { array }\end{array}$ & $2 \lambda / D$ & $2 \lambda / D$ & 34 & 1 \\
$\begin{array}{c}\text { Interfer. horn } \\
\text { array }\end{array}$ & $\lambda / 2 D$ & $2 \lambda / d$ & 8.6 & 16 \\
\hline
\end{tabular}

To achieve the same angular resolution each instrument allows different amounts of throughput (number of modes) and requires different aperture diameters, $D$. For the Gregorian the edge taper on the primary mirror illumination is assumed to be $-40 \mathrm{~dB}$, the diameter of the FOV is given in degrees and the number of modes is approximately [FOV/(angular resolution) $]^{2}$, assuming all the modes reaching the focal plane are coupled to detectors. For the imaging horn array, the horn diameter $=D$. For the interferometric horn array, $D=B$, the diameter of a close-packed array of horns, each of diameter $d$, and the number of modes is given by the number of horns $\sim(D / d)^{2}$. In the last three columns, for all cases, the angular resolution $=1^{\circ}$ and $\lambda=3 \mathrm{~mm}$.

Imaging instruments for CMB polarization measure the power in each linear polarization on separate bolometers and then form the difference of the two signals to determine the linear polarization. This approach requires careful matching of the bolometers. Moreover, if the signals being differenced come from two different antennas, then the beam patterns and pointing of the two antennas must coincide precisely. Any mismatch converts power from the total intensity into a spurious polarization signal $(\mathrm{Hu}$ et al., 2003). In an interferometer, differences in antenna patterns for the different horns do not couple intensity to polarization in this way (see Section 4).

An interferometer measures the Stokes parameters by correlating the components of the electric field captured by each antenna with the components from all of the other antennas. If the output of each antenna is split into $E_{x}$ and $E_{y}$ by an orthomode transducer (OMT), on the baseline formed by two antennas, 1 and 2 , the interferometer's correlators measure $\left\langle E_{1 x} E_{2 x}\right\rangle,\left\langle E_{1 y} E_{2 y}\right\rangle,\left\langle E_{1 x} E_{2 y}\right\rangle$, and $\left\langle E_{1 y} E_{2 x}\right\rangle$. The first two are used to determine $I$ and the latter two measure $U$. Rotating the instrument allows a measurement of $Q$. Stokes $V$ can be recovered in a similar manner but is expected to be zero for the CMB. Alternatively, the antenna outputs can be separated into left- and right-circular polarization components by a combination of an OMT and a polarizer. Correlating these signals also allows recovery of all four Stokes parameters. DASI uses a switchable polarizer to accomplish this (Leitch et al., 2002).

Separation of $E$ and $B$ modes. A significant challenge in $\mathrm{CMB}$ polarization measurements is separation of the very weak $B$ modes from the much stronger $E$ modes. Unless a full-sky map (an impossibility because of Galactic cuts) is made with infinite angular resolution the two modes "leak" into each other (Lewis et al., 2002; Bunn, 2003). It has been shown (Park et al., 2003; Park and Ng, 2004), however, that an interferometer can separate the $E$ and $B$ modes more cleanly than can an imaging experiment, 
although detailed calculations of this advantage in realistic simulations remain to be done.

\section{Systematic effects}

Hu et al. (2003) have reviewed systematic effects relevant to CMB polarization measurements, mainly in the context of imaging instruments. Bunn (submitted for publication) performs similar calculations for interferometers. Table 3 outlines a variety of systematic errors and how they can be managed in imaging and interferometric instruments. The relative importance of these effects is quite different in interferometric systems: some sources of systematic error in imaging systems are dramatically reduced in interferometers. As an example we consider the effects of pointing errors and mismatched antenna patterns.

In a traditional imaging system, the Stokes parameters $Q$ and $U$ are determined by subtracting the intensities of two different polarizations. For example, $Q$ might be measured by splitting the incoming radiation into $x$ and $y$ polarizations, determining the intensities $I_{x}$ and $I_{y}$ of the two polarizations, and subtracting. In such an experiment, any mismatch in the beam patterns used to determine $I_{x}$ and $I_{y}$ (including differential pointing errors as well as different beam shapes) will cause leakage from total power $(T)$ into polarization $(Q, U)$.

In an interferometer, the signals are combined before squaring to get intensities. In such a system, mismatched beams do not lead to leakage from temperature into polarization. Suppose that the signal entering each horn of an interferometer is split into horizontal and vertical polarizations. Working in the flat-sky approximation, let $E_{i x}(r)$ and $E_{i y}(r)$ stand for the $x$ and $y$ components of the electric field of the radiation entering the $i$ th horn from position $r$ on the sky. The signals coming out of each horn are averages of the incoming electric fields weighted by some antenna patterns $G_{i(x, y)}(r)$.

In an interferometer, these signals are multiplied together to obtain a visibility. To measure the Stokes parameter $U$, for example, we would multiply the $x$ signal from horn $i$ with the $y$ signal from horn $j$ to obtain the visibility

$V_{i j}^{U}=\int \mathrm{d}^{2} r_{1} \mathrm{~d}^{2} r_{2} G_{i x}\left(r_{1}\right) G_{j y}\left(r_{2}\right)\left\langle E_{i x}\left(r_{1}\right) E_{j y}^{*}\left(r_{2}\right)\right\rangle$.

The angle brackets denote a time average. The electric fields due to radiation coming from two different points on the sky are uncorrelated, and the product of $x$ and $y$ components of the electric field gives the Stokes $U$ parameter:

$\left\langle E_{i x}\left(r_{1}\right) E_{j y}^{*}\left(r_{2}\right)\right\rangle=U\left(r_{1}\right) \mathrm{e}^{2 \pi i u \cdot r_{1}} \delta\left(r_{1}-r_{2}\right)$,

so the visibility is

$V_{i j}^{U}=\int \mathrm{d}^{2} r G_{i x}(r) G_{j y}(r) U(r) \mathrm{e}^{2 \pi i u \cdot r}$.

Note that the visibility $V_{i j}^{U}$ does not contain any contribution from the total intensity (Stokes $I$ ), even if the two antenna patterns are different. This means that differential pointing errors and different beam shapes for different antennas do not cause leakage from $T$ into $E$ and $B$. Antenna pattern differences do cause distortion of the observed polarization field, so errors in modeling beam shapes and pointing may cause mixing between $E$ and $B$.

Coupling between intensity and polarization will arise if the beams have cross-polar contributions. In that case, the visibility $V_{i j}^{U}$, which is supposed to be sensitive to just polarization, will contain contributions proportional to $\left\langle E_{x} E_{x}^{*}\right\rangle$ and $\left\langle E_{y} E_{y}^{*}\right\rangle$, to which Stokes $I$ does contribute.

The same considerations apply if the incoming radiation is split into circular rather than linear polarization states. The visibility $V_{i j}^{\mathrm{RL}}$, obtained by interfering the right-circularly-polarized signal entering horn $i$ with the left-circularly-polarized signal entering horn $j$, contains only contributions from $Q$ and $U$ if the beams are co-polar, even if the two horns have different beams. Again, cross-polarity induces leakage from intensity into polarization.

In short, in an interferometer, beam mismatches are less of a worry than cross-polar contributions. The reverse is true for an imaging system.

Table 3

A comparison of systematic effects

\begin{tabular}{|c|c|c|}
\hline Systematic effect & Imaging system solution & Interferometer solution \\
\hline Cross-polar beam response & Instrument rotation and correction in analysis & Instrument rotation and non-reflective optics \\
\hline Beam ellipticity & Instrument rotation and small beamwidth & No $T$ to $E$ and $B$ leakage from beams; inst. rot'n \\
\hline Polarized sidelobes & Correction in analysis & Correction in analysis \\
\hline Instrumental polarization & Rotation of instrument and correction in analysis & Clean, non-reflective optics \\
\hline Polarization angle & Construction and characterization & $\begin{array}{l}\text { No } T \text { to } E \text { and } B \text { leakage from beams; construction and } \\
\text { characterization }\end{array}$ \\
\hline Relative pointing & $\begin{array}{l}\text { Rotation of instrument and dual polarization } \\
\text { pixels }\end{array}$ & No $T$ to $E$ and $B$ leakage from beams; inst. rot'n \\
\hline Relative calibration & $\begin{array}{l}\text { Measure calibration using temperature } \\
\text { anisotropies }\end{array}$ & Detector comparison not req'd for mapping or measuring $Q$ and $U$ \\
\hline Relative calibration drift & Control scan-synchronous drift to $10^{-9}$ level & All signals on all detectors \\
\hline Optics temperature drifts & Cool optics to $\sim 3 \mathrm{~K}$ stabilize to $<\mu \mathrm{K}$ & No reflective optics \\
\hline $1 / f$ noise in detectors & Scanning strategy and phase modulation/lock-in & Instant. measurement of power spectrum without scanning \\
\hline Astrophysical foregrounds & Multiple frequency bands & Multiple frequency bands \\
\hline
\end{tabular}




\section{The adding interferometer}

In a simple 2-element radio interferometer, signals from two telescopes aimed at the same point in the sky are correlated so that the sky temperature is sampled with an interference pattern with a single spatial frequency. The output of the multiplying interferometer is the visibility (defined in the last section).

With more antennas these same correlations are performed along each baseline. To recover the full phase information, complex correlators are used to measure simultaneously both the in-phase and quadrature-phase components of the visibility.

In interferometers that use incoherent detectors, such as optical interferometers or EPIC and MBI, the electric field wavefronts from two antennas are added and then squared in a detector - an "adding" interferometer as opposed to a "multiplying" interferometer (Rohlfs and Wilson, 2004) (see Fig. 1). The result is a constant term proportional to the intensity plus an interference term. The constant term is an offset that is removed by phase-modulating one of the signals. Phase-sensitive detection at the modulation frequency recovers both the in-phase and quadrature-phase interference terms and reduces susceptibility to low-frequency drifts $(1 / f$ noise $)$ in the bolometer and readout electronics. The adding interferometer recovers the same visibility as a multiplying interferometer.

In an interferometer with an array of $N>2$ antennas, the signals are combined in such a way that interference fringes are measured for all possible baselines $(N(N-1) / 2$ antenna pairs). This combination can occur in two different ways: pairwise combination or Butler (or Fizeau) combination (Zmuidzinas, 2003). Pairwise combination involves splitting the power from each of the $N$ antennas in the array $N-1$

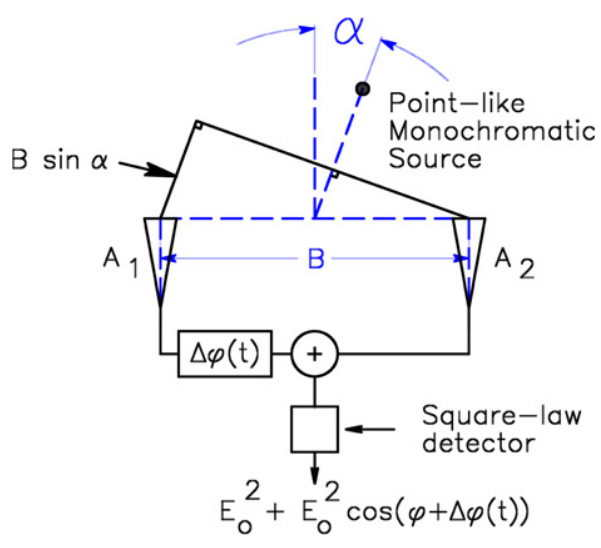

Fig. 1. Adding interferometer. At antenna $A_{2}$ the electric field is $E_{0}$, and at $A_{1}$ it is $E_{0} \mathrm{e}^{\mathrm{i} \phi}$, where $\phi=k B \sin \alpha$ and $k=2 \pi / \lambda . B$ is the length of the baseline, and $\alpha$ is the angle of the source with respect to the symmetry axis of the baseline, as shown. (For simplicity consider only one wavelength, $\lambda$, and ignore time dependent factors.) In a multiplying interferometer the inphase output of the correlator is proportional to $E_{0}^{2} \cos \phi$. For the adding interferometer, the output is proportional to $E_{0}^{2}+E_{0}^{2} \cos (\phi+\Delta \phi(t))$. Modulation of $\Delta \phi(t)$ allows the recovery of the interference term, $E_{0}^{2} \cos \phi$, which is proportional to the visibility of the baseline. ways, adding the signals in a pairwise fashion, and then squaring the signals and separating out the interference term as described above. In optical systems this approach is analogous to Michelson interferometry. In Butler combination the signals from each of the antennas are split and then combined in such a way that linear combinations of all the antenna signals are formed at each of the outputs of the combiner (Fig. 2). To allow all the Stokes parameters to be determined simultaneously, orthomode transducers (OMTs) are inserted after corrugated horn antennas. In this case, the Butler combiner delivers the signals from $2 N$ antenna outputs to $2 N$ detectors. Each detector squares these amplitudes, creating interference signals from all baselines simultaneously on each detector. Effectively, the signals from all baselines are multiplexed onto each of the $N$ detectors. Only $2 N$ detectors are required, rather than the $2 N(2 N-1) / 2$ detectors required for pairwise combination. Butler combiners are commonly used for phased array antennas with coherent systems using either waveguide or coaxial techniques. The optical analog is Fizeau combination, which is typically used for incoherent systems at optical wavelengths. We have developed both Butler and Fizeau approaches and have decided to concentrate on the Fizeau method because of its relative simplicity and low-loss. However, in a coherent system, with amplifiers, the Butler approach is still an attractive option for forming a large$N$ interferometer.

Fig. 3 shows a possible configuration for a interferometric module for EPIC. The array views the sky through a close-packed cluster of corrugated horn antennas. The two polarizations (either linear or circular) are split by an

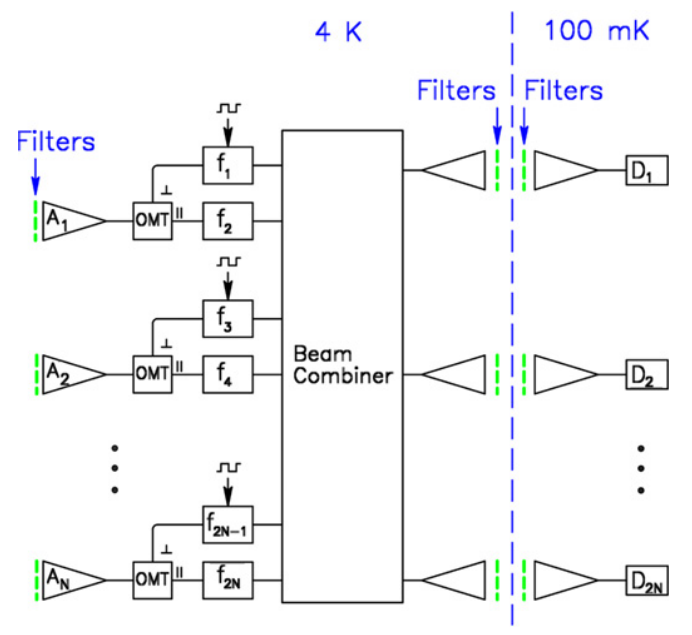

Fig. 2. Block diagram of EPIC. Light enters the instrument from the left. Each phase switch is modulated in a sequence that allows recovery of the interference terms (visibilities) by phase-sensitive detection at the detectors. The signals are mixed in the beam combiner and detected on cold bolometers at the right. The beam combiner can be implemented either using guided waves (Butler combiner, as shown here) or quasioptically (Fizeau combiner, see below). The triangles represent corrugated conical horn antennas, which connect through transitions to rectangular waveguide. Orthomode transducers (OMTs) allow all the Stokes parameters to be determined simultaneously. 


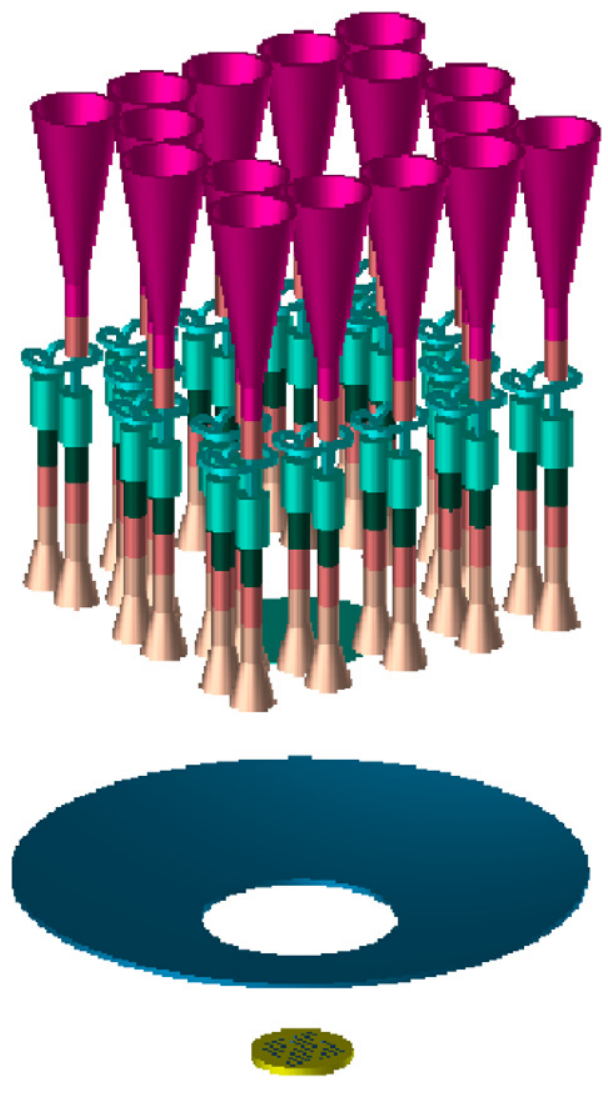

Fig. 3. A three-dimensional view of 16 corrugated horn antennas arranged in a close packed array illuminating a Fizeau combiner. The detector array sits behind the primary mirror of the beam combiner. Note that the distances between the antennas, primary mirror and detector array are not to scale. EPIC could be made of a cluster of these fundamental modules, with multiple copies operating at frequencies from 30 to $300 \mathrm{GHz}$.

ortho-mode transducer and individually phase-modulated (Fig. 4). The beams are then combined with a Fizeau combiner in the form of a cold, compact, on-axis Cassegrain telescope. Interference fringes formed by the various antenna baselines appear on the bolometer array in the focal plane of the telescope. The superimposed fringes are separated from each other using a phase modulation sequence that uniquely encodes each visibility (Fig. 5).

\section{EIP design}

The science that the EIP will discover is determined by the range of angular scales probed by the instrument. $W M A P$, for example, has demonstrated the interesting nature of the lowest- $l(2,3,4,5)$ multipoles in the $T T$ and $T E$ power spectra. At low $l$ the $B B$ polarization power spectrum is most sensitive to the primordial gravitational wave signal. At larger $l$ the $B B$ polarization power spectrum measures gravitational lensing of the CMB. The EIP will study the polarization from the lowest- $l$ multipoles to multipoles high enough to measure the lensing signal. Whether the EIP is an interferometer or an imaging system, the con-

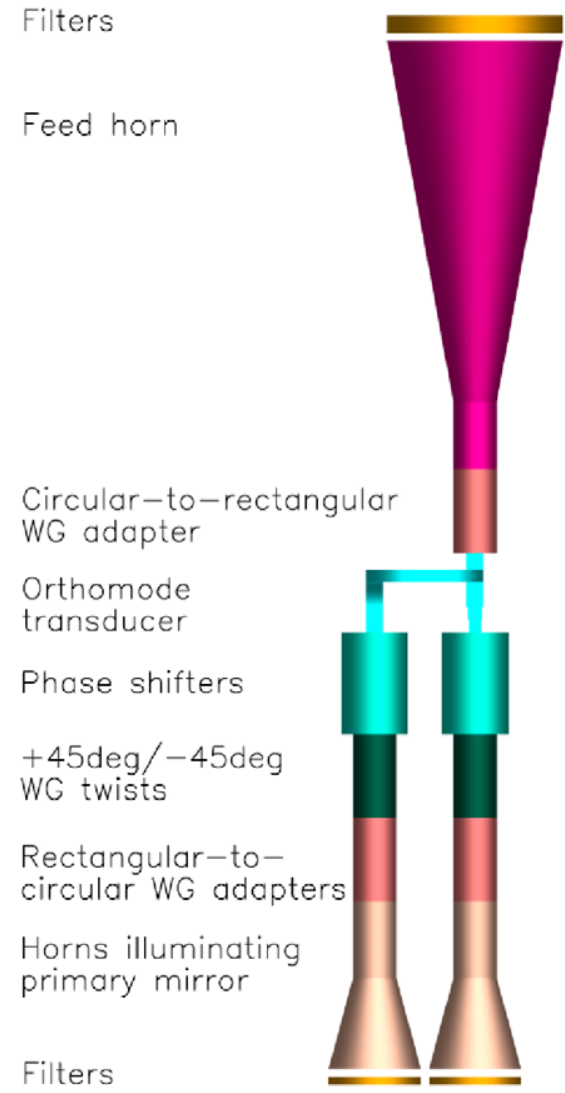

Fig. 4. Input unit (IU) of the EPIC interferometer. The two polarizations are separated using an orthomode transducer and are rotated in waveguide (WG) so that the two polarization vectors are aligned. A $\pm 90^{\circ}$ phase modulation is introduced in one of the arms and the two signals are directed at the Fizeau combiner. The interference of the two signals from an IU results in a correlation receiver, instantaneously sensitive to the Stokes U parameter. The interference of signals from different IUs results in an interferometer.

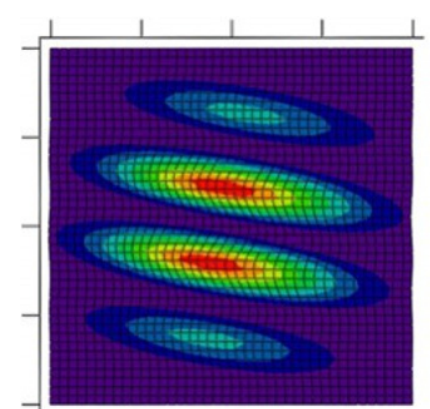

a

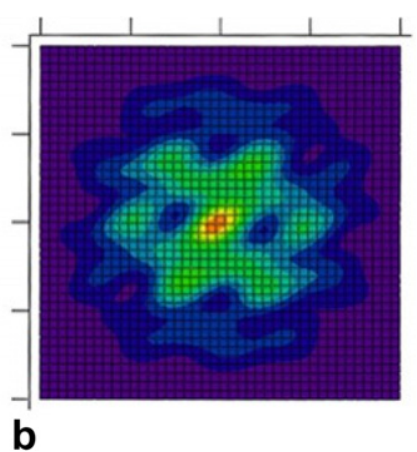

b
Fig. 5. (a) Simulation of fringe patterns formed in the focal plane of the Fizeau beam combiner from a single baseline. (b) Superposition of fringes from 6 baselines (as expected in MBI). Fringes are separated by the phase modulation sequence.

figuration will have to be optimized to measure this range of multipoles with appropriate signal-to-noise.

In the case of an interferometric design, the main configuration issues are the field of view of the horns and the placement of antennas to get optimal coverage of the $u v$ plane. In an imaging system, the main configuration issues 
are the angular resolution and field of view and the number and placement of detectors in the focal plane. In both cases, minimization of systematic effects depends upon a careful choice of scan strategy.

The EPIC mission concept includes multiple closepacked arrays of horn antennas that are co-aligned and pointed directly at the sky, with no intervening lenses or reflectors. Each array is configured as an adding interferometer using the beam combination scheme of Fig. 3. The interferometer measures the visibilities from all baselines in the array. In addition, the phase modulators can be operated in such a way that the signals from each antenna interfere with themselves. In this mode the system acts as an array of correlation polarimeters, sensitive to $Q$ and $U$ averaged over a single antenna beam. The correlation polarimeter mode is used to measure the lowest spherical harmonics, while the interferometric mode recovers the higher-order multipoles. Both of these modes can operate simultaneously.

In an interferometer, each individual pointing covers a large sky area and samples many different baselines simultaneously, potentially reducing systematic errors in mapmaking. We are studying how to combine the visibilities measured with successive pointings of the instrument over large portions of the sky-a process called mosaicking (Bunn and White, in press). The relatively simple configuration of the EPIC instrument may allow for an additional degree of freedom in the scan using rotation of the instrument. If the low- $l$ modes are recovered by using the instrument in a correlation receiver configuration, then scan-strategy issues similar to those of an imaging system may arise.

Fig. 6 displays the sensitivity for one possible configuration under study for EPIC. There is a total of 60 arrays, each including 16 close-packed corrugated horn antennas, for a total of 960 horns. Each horn has a beam width of $7^{\circ}$. Each array operates in wide bands $(\sim 20 \%)$ centered at frequencies between $\sim 30-300 \mathrm{GHz}$. There are 48 arrays sensitive to $90 \mathrm{GHz}$, the primary science channel. The other 12 arrays are for measuring and removing foregrounds; they are not included in the sensitivity estimate. The instrument and observing patterns have not been optimized. The exact band placements and number of bands will be chosen to optimize the removal of foreground contamination. The detectors are cold $(\sim 100 \mathrm{mK})$, background-limited superconducting transition-edge sensors (TES) read out by SQUID multiplexers. Based on our measurements, the instrument efficiency is taken to be $50 \%$. Emission from the cryostat window dominates the optical loading on the detectors. EPIC surveys the full sky with a combination of instrument rotation and precession.

\section{The millimeter-wave bolometric interferometer (MBI)}

The millimeter-wave bolometric interferometer is a ground-based instrument designed to demonstrate bolometric interferometry and to search for polarization. The first generation of this instrument, called MBI-4, has four input antennas. The input of MBI is similar to that of EPIC

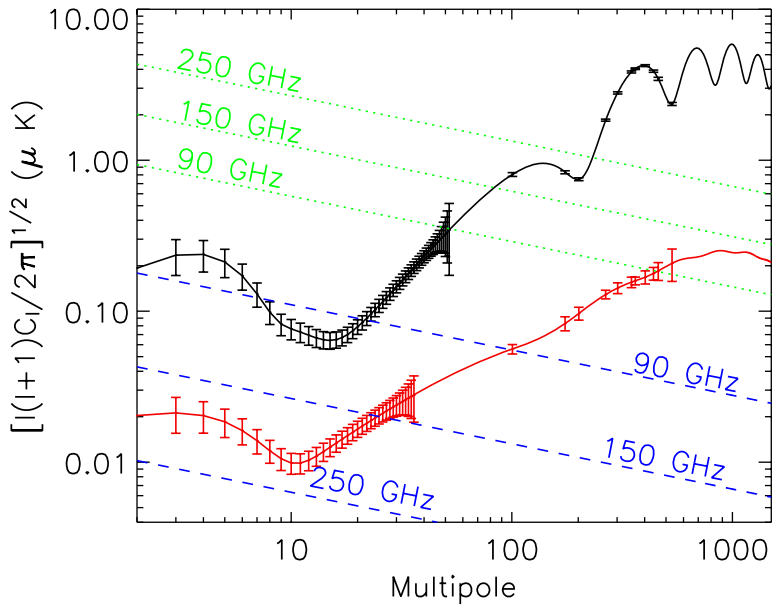

Fig. 6. Expected sensitivity of EPIC, a mission concept for the Einstein Inflation Probe, to $E$ (black) and $B$ (red) polarization. The power spectra are based on the best-fit model from WMAP (Spergel et al.). The tensorto-scalar ratio is taken to be 0.01 . Errors $(1 \sigma)$ assume one year of integration sampling the full sky uniformly. The estimates are representative of the capabilities of possible designs for the EIP. The configuration assumed here includes 960 feed horns, with 768 sensitive to $90 \mathrm{GHz}$, the primary science channel; the other 192 feed horns, sensitive to 150 and $250 \mathrm{GHz}$, are for measuring and removing foregrounds and are not included in this estimate. The dotted lines show the expected levels of polarized dust emission and the dashed lines show the expected levels of polarized synchrotron emission at 90,150 and $250 \mathrm{GHz}$ based on the WMAP results (Page et al.). EPIC operates both as an imaging instrument and an interferometer; low- $l$ points come from operating the interferometer as single-beam correlation radiometers while high- $l$ points come from operating the instrument as an interferometer (see Section 6). (For interpretation of the references to color in this figure legend, the reader is referred to the web version of this article.)

(Fig. 4), but in MBI each antenna selects a single linear polarization (there are no OMTs). As a result, MBI allows interference of signals from different antennas but does not have a correlation polarimeter mode of operation. The configuration of MBI-4 optics and cryostat is shown in Fig. 7. A photograph of the MBI-4 optics is shown in Fig. 8.

The cryostat is attached to an altitude-azimuth mount. This mount has a third axis to rotate the instrument about its optical axis. The cryostat uses 17 liters of liquid nitrogen and 25.7 liters of liquid helium and lasts for about $50 \mathrm{~h}$.

The feed horn configuration is chosen to provide uniform $u v$ coverage with $10^{\circ}$ step rotations of the instrument about its optical axis. The instrument is sensitive to $\mathrm{CMB}$ polarization fluctuations in the multipole range from $\ell=150$ to 270 . The "temperature" baselines (e.g. interference of $E_{i x}$ with $E_{j x}$ ) will be used to calibrate by comparison with the WMAP temperature maps.

The phase of each of the four inputs is sequentially modulated between $-90^{\circ}$ and $+90^{\circ}$ using ferrite-based modulators (Keating et al., in preparation) implemented in circular waveguide. The modulation rate is $\sim 10-100 \mathrm{~Hz}$ and the loss is $<1 \mathrm{~dB}$. The phase shifters dissipate negligible power, $\sim 1 \mathrm{~mW}$ each. Differential loss between the two phase states will produce an offset after demodulation of the detector signal, so the differential loss between the two phase states must be small. 


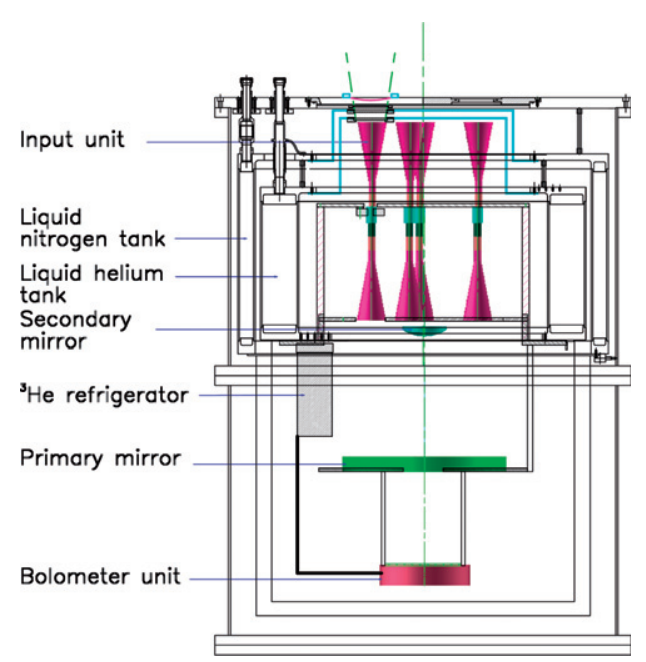

Fig. 7. The MBI-4 optics and cryostat. The four input units are visible at the top of the cryostat. The beams are combined with a Cassegrain telescope acting as a Fizeau combiner. The bolometer unit, cooled by the ${ }^{3} \mathrm{He}$ refrigerator, also contains filters. The cryostat uses both liquid nitrogen and liquid helium.

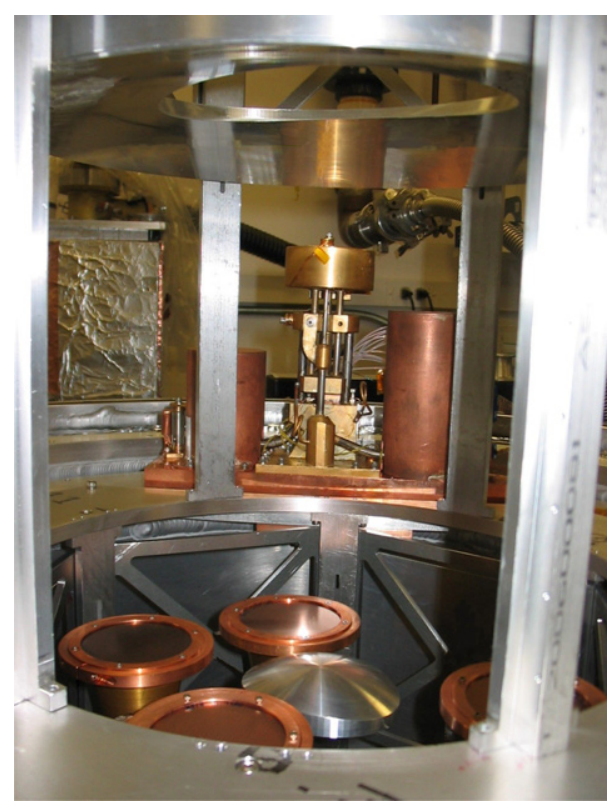

Fig. 8. MBI-4. In this view, the cryostat is upside down and light is entering from the bottom of the figure. At the bottom are the four input units and secondary mirror of the Fizeau combiner. At the top is the primary mirror of the Fizeau combiner. The bolometers are located above the primary mirror and are not visible. Visible at the back is the ${ }^{3} \mathrm{He}$ refrigerator.

The light is interfered on an array of 16 bolometers at the focal plane of the primary mirror. MBI-4 uses spiderweb bolometers, provided by JPL, with NTD germanium thermistors. The bolometers are coupled to the incoming radiation with conical horns; the horns form a hexagonally packed array. The bolometers and horns are cooled to $\sim 330 \mathrm{mK}$ with a ${ }^{3} \mathrm{He}$ refrigerator.
The ${ }^{3} \mathrm{He}$ refrigerator, manufactured by Simon Chase, lasts for at least $90 \mathrm{~h}$. The condensor is cooled by a selfcontained ${ }^{4} \mathrm{He}$ charcoal-pumped pot.

MBI-4 will be demonstrated at the Pine Bluff Observatory (PBO) near Madison, Wisconsin. Key tests include measuring the interferometric beam patterns, observing bright objects such as the moon, and during the winter, when atmospheric conditions are good, carrying out long integrations on test fields.

\section{Conclusion}

We have described a novel interferometric approach to measuring the faint polarization in the CMB. In this scheme we observe the sky directly with a close-packed array of circular corrugated horn antennas and combine the signals with a Fizeau combiner. This approach uses the aperture plane efficiently and provides an alternate way to control systematic effects compared to imaging instruments. It takes advantage of advances in the development of arrays of sensitive bolometric detectors for frequencies of interest to CMB measurements ( 30 to $300 \mathrm{GHz})$, but can also be used with coherent receivers. We gave an overview of the EPIC NASA mission concept study underway for the Einstein Inflation Probe as well as a review of the status of MBI, a ground-based demonstration of the technique.

\section{Acknowledgements}

The EPIC mission concept study is supported by NASA award NNG04GI15G. MBI is supported by NASA awards NAG5-12758 at Brown and NNG04GC13G at Wisconsin. P.T. acknowledges support from the UW Vilas Associates Program. This work was performed under the auspices of the U.S. Department of Energy by University of California, Lawrence Livermore National Laboratory under Contract W-7405-Eng-48.

\section{References}

Baker, J.C., Grainge, K., Hobson, M.P., Jones, M.E., Kneissl, R., Lasenby, A.N., O’Sullivan, C.M.M., Pooley, G., Rocha, G., Saunders, R., Scott, P.F., Waldram, E.M., 1999. Detection of cosmic microwave background structure in a second field with the Cosmic Anisotropy Telescope. MNRAS 308, 1173-1178.

Birkinshaw, M., 1999. The Sunyaev-Zel'dovich effect. PhR 310, 97-195.

Bock, J., Church, S., Devlin, M., Hinshaw, G., Lange, A., Lee, A., Page, L., Partridge, B., Ruhl, J., Tegmark, M., Timbie, P., Weiss, R., Winstein, B., Zaldarriaga, M. Task force on cosmic microwave background research, ArXiv Astrophysics e-prints.

Bunn, E.F., 2003. Separating E from B. NewAR 47, 987-994.

Bunn, E.F., Systematic errors in microwave background interferometry. PhRvD (submitted for publication).

Bunn, E.F., White, M. Mosaicking with cosmic microwave background interferometers. ApJ (in press).

Carlstrom, J.E., Holder, G.P., Reese, E.D., 2002. Cosmology with the Sunyaev-Zel'dovich Effect. Ann. Rev. Ast. Astrop. 40, 643-680.

Carretti, E., Tascone, R., Cortiglioni, S., Monari, J., Orsini, M., 2001. Limits due to instrumental polarisation in $\mathrm{CMB}$ experiments at microwave wavelengths. NewA 6, 173-187. 
Carretti, E., Cortiglioni, S., Sbarra, C., Tascone, R., 2004. Antenna instrumental polarization and its effects on E- and B-modes for CMBP observations. A\&A 420, 437-445.

Cartwright, J.K., Pearson, T.J., Readhead, A.C.S., Shepherd, M.C., Sievers, J.L., Taylor, G.B., 2005. Limits on the polarization of the cosmic microwave background radiation at multipoles up to $1 \sim 2000$. ApJ 623, 11-16.

Dawson, K.S., Holzapfel, W.L., Carlstrom, J.E., Joy, M., LaRoque, S.J., Reese, E.D., 2001. A preliminary detection of arcminute-scale cosmic microwave background anisotropy with the BIMA array. ApJL 553, L1-L4.

Dickinson, C., Battye, R.A., Carreira, P., Cleary, K., Davies, R.D., Davis, R.J., Genova-Santos, R., Grainge, K., Gutiérrez, C.M., Hafez, Y.A., Hobson, M.P., Jones, M.E., Kneissl, R., Lancaster, K., Lasenby, A., Leahy, J.P., Maisinger, K., Ödman, C., Pooley, G., Rajguru, N., Rebolo, R., Rubiño-Martin, J.A., Saunders, R.D.E., Savage, R.S., Scaife, A., Scott, P.F., Slosar, A., Sosa Molina, P., Taylor, A.C., Titterington, D., Waldram, E., Watson, R.A., Wilkinson, A., 2004. High-sensitivity measurements of the cosmic microwave background power spectrum with the extended Very Small Array. MNRAS 353, 732-746.

Fowler, J.W., Doriese, W.B., Marriage, T.A., Tran, H.T., Aboobaker, A.M., Dumont, C., Halpern, M., Kermish, Z.D., Loh, Y.-S., Page, L.A., Staggs, S.T., Wesley, D.H., 2005. Cosmic microwave background observations with a compact heterogeneous $150 \mathrm{GHz}$ interferometer in Chile. ApJSS 156, 1-11.

Goldsmith, P.F., 1998. Quasioptical Systems. IEEE Press.

Halverson, N.W., Leitch, E.M., Pryke, C., Kovac, J., Carlstrom, J.E., Holzapfel, W.L., Dragovan, M., Cartwright, J.K., Mason, B.S., Padin, S., Pearson, T.J., Readhead, A.C.S., Shepherd, M.C., 2002. Degree angular scale interferometer first results: a measurement of the cosmic microwave background angular power spectrum. ApJ 568, 38-45.

Hanany, S., Marrone, D.P., 2002. Comparison of designs of off-axis Gregorian telescopes for millimeter-wave large focal-plane arrays. AO 41, 4666-4670.

Hu, W., Hedman, M.M., Zaldarriaga, M., 2003. Benchmark parameters for CMB polarization experiments. PhRvD 67 (4), 043004.

Jones, M.E., Edge, A.C., Grainge, K., Grainger, W.F., Kneissl, R., Pooley, G.G., Saunders, R., Miyoshi, S.J., Tsuruta, T., Yamashita, K., Tawara, Y., Furuzawa, A., Harada, A., Hatsukade, I., 2005. $H_{0}$ from an orientation-unbiased sample of Sunyaev-Zel'dovich and X-ray clusters. MNRAS 357, 518-526.

Keating, B., Bock, J., Erickson, N., Gaier, T., Lange, A., Rorie, J., Siegal, D., Wollack, E., Linear polarization modulator for mm-wavelengths, in preparation.

Knox, L., Song, Y.-S., 2002. Limit on the detectability of the energy scale of inflation. PhRvL 89 (1), 011303.

Korotkov, A., Kim, J., Tucker, G., Gault, A., Hyland, P., Malu, S., Timbie, P., Bunn, E., Bierman, E., Keating, B., Murphy, A., O'Sullivan, C., Ade, P., Calderon, C., Piccirillo, L., 2006. The millimeter-wave bolometric interferometer. Proceedings of the SPIE, vol. 6272, 62750X-1.

Kovac, J.M., Leitch, E.M., Pryke, C., Carlstrom, J.E., Halverson, N.W., Holzapfel, W.L., 2002. Detection of polarization in the cosmic microwave background using DASI. Nature 420, 772-787.

Leitch, E.M., Readhead, A.C.S., Pearson, T.J., Myers, S.T., Gulkis, S., Lawrence, C.R., 2000. A measurement of anisotropy in the cosmic microwave background on $7^{\prime}-22^{\prime}$ scales. ApJ 532, 37-56.

Leitch, E.M., Kovac, J.M., Pryke, C., Carlstrom, J.E., Halverson, N.W., Holzapfel, W.L., Dragovan, M., Reddall, B., Sandberg, E.S., 2002. Measurement of polarization with the Degree Angular Scale Interferometer. Nature 420, 763-771.

Leitch, E.M., Kovac, J.M., Halverson, N.W., Carlstrom, J.E., Pryke, C., Smith, M.W.E., 2005. Degree angular scale interferometer 3 year cosmic microwave background polarization results. ApJ 624, $10-20$.

Lewis, A., Challinor, A., Turok, N., 2002. Analysis of CMB polarization on an incomplete sky. PhRvD 65 (2), 023505.
Loh, M., Carlstrom, J.E., Cartwright, J.K., Greer, C., Hawkins, D., Hennessy, R., Joy, M., Lamb, J., Leitch, E., Miller, A., Mroczkowski, T., Muchovej, S., Pryke, C., Reddall, B., Richardson, G., Runyan, M., Sharp, M., Woody, D., 2005. The Sunyaev-Zel'dovich Array. American Astronomical Society Meeting Abstracts 207, p. 41.01.

MacTavish, C.J., Ade, P.A.R., Bock, J.J., Bond, J.R., Borrill, J., Boscaleri, A., Cabella, P., Contaldi, C.R., Crill, B.P., de Bernardis, P., De Gasperis, G., de Oliveira-Costa, A., De Troia, G., Di Stefano, G., Hivon, E., Jaffe, A.H., Jones, W.C., Kisner, T.S., Lange, A.E., Lewis, A.M., Masi, S., Mauskopf, P.D., Melchiorri, A., Montroy, T.E., Natoli, P., Netterfield, C.B., Pascale, E., Piacentini, F., Pogosyan, D., Polenta, G., Prunet, S., Ricciardi, S., Romeo, G., Ruhl, J.E., Santini, P., Tegmark. M., Veneziani, M., Vittorio, N. Cosmological Parameters from the 2003 flight of BOOMERANG, ArXiv Astrophysics e-prints.

Page, L., Hinshaw, G., Komatsu, E., Nolta, M.R., Spergel, D.N., Bennett, C.L., Barnes, C., Bean, R., Dore', O., Halpern, M., Hill, R.S., Jarosik, N., Kogut, A., Limon, M., Meyer, S.S., Odegard, N., Peiris, H.V., Tucker, G.S., Verde, L., Weiland, J.L., Wollack, E., Wright, E.L. Three year Wilkinson Microwave Anisotropy Probe (WMAP) observations: polarization analysis, ArXiv Astrophysics e-prints.

Park, C.-G., Ng, K.-W., 2004. E/B separation in cosmic microwave background interferometry. ApJ 609, 15-21.

Park, C.-G., Ng, K.-W., Park, C., Liu, G.-C., Umetsu, K., 2003. Observational strategies of cosmic microwave background temperature and polarization interferometry experiments. ApJ 589, 67-81.

Partridge, R.B., Richards, E.A., Fomalont, E.B., Kellermann, K.I., Windhorst, R.A., 1997. Small-scale cosmic microwave background observations at $8.4 \mathrm{GHz}$. ApJ 483, 38.

Readhead, A.C.S., Mason, B.S., Contaldi, C.R., Pearson, T.J., Bond, J.R., Myers, S.T., Padin, S., Sievers, J.L., Cartwright, J.K., Shepherd, M.C., Pogosyan, D., Prunet, S., Altamirano, P., Bustos, R., Bronfman, L., Casassus, S., Holzapfel, W.L., May, J., Pen, U.-L., Torres, S., Udomprasert, P.S., 2004. Extended mosaic observations with the cosmic background imager. ApJ 609, 498-512.

Readhead, A.C.S., Myers, S.T., Pearson, T.J., Sievers, J.L., Mason, B.S., Contaldi, C.R., Bond, J.R., Bustos, R., Altamirano, P., Achermann, C., Bronfman, L., Carlstrom, J.E., Cartwright, J.K., Casassus, S., Dickinson, C., Holzapfel, W.L., Kovac, J.M., Leitch, E.M., May, J., Padin, S., Pogosyan, D., Pospieszalski, M., Pryke, C., Reeves, R., Shepherd, M.C., Torres, S., 2004. Polarization observations with the cosmic background imager. Science 306, 836-844.

Rohlfs, K., Wilson, T.L., 2004. Tools of Radio Astronomy. Springer, Berlin.

Spergel, D.N., Bean, R., Dore', O., Nolta, M.R., Bennett, C.L., Hinshaw, G., Jarosik, N., Komatsu, E., Page, L., Peiris, H.V., Verde, L., Barnes, C., Halpern, M., Hill, R.S., Kogut, A., Limon, M., Meyer, S.S., Odegard, N., Tucker,G.S., Weiland, J.L., Wollack, E., Wright, E.L. Wilkinson Microwave Anisotropy Probe (WMAP) three year results: implications for cosmology, ArXiv Astrophysics e-prints.

Subrahmanyan, R., Kesteven, M.J., Ekers, R.D., Sinclair, M., Silk, J., 2000. An Australia telescope survey for CMB anisotropies. MNRAS $315,808-822$.

Timbie, P.T., Wilkinson, D.T., 1990. A search for anisotropy in the cosmic microwave radiation at medium angular scales. ApJ 353, 140-144.

White, M., Carlstrom, J.E., Dragovan, M., Holzapfel, W.L., 1999. Interferometric observation of cosmic microwave background anisotropies. ApJ 514, 12-24.

Woody, D.P., Beasley, A.J., Bolatto, A.D., Carlstrom, J.E., Harris, A., Hawkins, D.W., Lamb, J., Looney, L., Mundy, L.G., Plambeck, R.L., Scott, S., Wright, M., 2004. CARMA: a new heterogeneous millimeterwave interferometer. In: Zmuidzinas, J., Holland, W.S., Withington, S. (Eds.), Astronomical Structures and Mechanisms Technology. Edited by Antebi, Joseph; Lemke, Dietrich. Proceedings of the SPIE, vol. 5498, 30-41.

Zmuidzinas, J., 2003. Cramér-Rao sensitivity limits for astronomical instruments: implications for interferometer design. Opt. Soc. Am. J. A $20,218-233$. 